\title{
REVISIONES
}

\section{Algunos códigos curriculares de la actual enseñanza básica chilena}

\author{
Curricular Codes in Current Chilean Elementary Education
}

Alguns códigos curriculares do atual ensino básico chileno

\author{
Luis Manríquez Pantoja. ${ }^{a}$
}

${ }^{a}$ Facultad de Educación, Universidad de Antofagasta, Chile. Fono: 55637840.

Correo electrónico: 1manriquez@uantof.cl

\section{RESUMEN}

Se caracterizan las bases curriculares vigentes para la Enseñanza Básica chilena, describiendo detalladamente el currículum centrado en el aprendizaje que subyace en el marco curricular y la problemática que ello implica.

Palabras clave: modelo curricular, estrategias cognitivas, estrategias metacognitivas, modelo conceptual, competencia, capacidad, habilidades.

\section{ABSTRACT}

This paper characterizes the current curricular foundations for elementary education in Chile, describing, in detail, the curriculum focused on learning underlying the curriculum framework and the problems involved.

Key words: curricular model, cognitive strategies, metacognitive strategies, conceptual model, competence, ability, skills.

\section{RESUMO}

Caracterizou-se a atual base curricular para a Educação Básica do Chile, descrevendo detalhadamente o currículo, centrando-o na aprendizagem subjacente à estrutura curricular e nos problemas envolvidos.

Palavras chave: modelo de currículo, estratégias cognitivas, estratégias metacognitivas, modelo conceitual, competência, capacidade, habilidades.

\section{INTRODUCCIÓN}

2013 podría considerarse como un año especial en el área del currículum de Enseñanza Básica, debido a que corresponde aplicar integralmente el nuevo marco curricular (también denominado Bases Curriculares) previsto por la Ley General de Educación (LGE), lo que cierra un proceso que se inició en 2009, cuando las autoridades de la época finalizan la tramitación de dos instancias legales, relacionadas directamente con el currículum chileno:

a) En agosto se publica para la Enseñanza Básica el Decreto Supremo 0256, el que modifica sustancialmente el D.S. N 40 de 1996 correspondiente al marco curricular proveniente de la Ley Orgánica Constitucional de Enseñanza, más conocida como "LOCE" y que el Ministerio de Educación (MINEDUC) la denomina "Ajuste Curricular", el que debía entrar en vigencia desde quinto a octavo básico en el año 2010, y en el año 2011 se debía incorporar desde primero a cuarto básico. 
b) En septiembre se promulga la ley 20.370, Ley General de Educación (LGE), cuerpo legal que introduce tres cambios cruciales al currículum chileno:

- Acorta la Enseñanza Básica a seis años.

- Introduce un nuevo cambio de nomenclatura (ahora se habla de Objetivos de Aprendizaje, nuevamente de asignaturas en vez de sectores o subsectores de aprendizaje, lo que obliga a generar un nuevo marco curricular, tanto en la Enseñanza Básica como en la Educación Media). El presente trabajo se refiere al Currículum de Enseñanza Básica.

Estos eventos aparentemente inocuos e inconexos, necesariamente introducen tensión el quehacer docente y, de alguna manera, podrían influir negativamente en los resultados académicos tan frecuentemente criticados, situación a la que pretendemos aportar elementos de reflexión.

El ajuste curricular, tal como se dijo, se debería haber iniciado en el segundo ciclo básico en 2010 según el artículo $2^{\circ}$ del citado decreto 256 (MINEDUC, 2009: vii), sin embargo, asumidas las nuevas autoridades educacionales debido al cambio de Gobierno, y como consecuencia del sismo del 27 de febrero de 2010 que afectó gravemente al centro de nuestro país, se envía un oficio ${ }^{1}$ postergando por un año la implementación del ajuste para el segundo ciclo, implicando de inmediato también la postergación por un año para el primer ciclo básico (2012 en vez de 2011). Nos imaginamos que a lo menos se generaron desajustes en la correlación de contenidos de los libros de texto (que se proyectan con tres años de anticipación) y los tópicos de las pruebas SIMCE, al margen del despliegue que el propio Ministerio de Educación había realizado para promover el Ajuste Curricular.

La situación se hace más compleja cuando las autoridades educacionales (del Gobierno del Presidente Piñera), haciendo uso de sus facultades legales, presentan ante el Consejo Nacional de Educación el Marco Curricular para la Enseñanza Básica (ahora de $1^{\circ}$ a $6^{\circ}$ Básico) asociada a la LGE, entidad que en septiembre del mismo año rechaza la propuesta con la excepción de Matemática e Inglés, lo que finalmente es aprobado en una segunda presentación, cuestión que permite promulgar las nuevas Bases Curriculares para la Enseñanza Básica $\left(1^{\circ}\right.$ a $\left.6^{\circ}\right)$ según el Decreto Supremo $N^{\circ} 439^{2}$.

Esta decisión implica que en el 2012 se aplican las Bases Curriculares de la LGE desde $1^{\circ}$ a $3^{\circ}$ Básico -ahora Primer Ciclo Básico según la nueva estructura-, quedando formalmente sin Marco Curricular $4^{\circ}$ Básico, el que queda sujeto a flexibilidad curricular como lo indica un instructivo ministerial (MINEDUC, 2012a). Está claro que en este primer ciclo, el Ajuste Curricular programado y difundido nunca fue aplicado. Una segunda implicancia es que el año 2012 conviven dos Marcos Curriculares, el de la LGE para primer ciclo y el de la LOCE desde $6^{\circ}$ a $8^{\circ}$ Básico.

Una tercera y última implicancia es que el Marco Curricular de $7^{\circ}$ y $8^{\circ}$ Básico continuará bajo las Bases Curriculares de la LOCE -Ajuste Curricular- conforme a la LGE hasta el año 2017, momento en que estos dos grados pasarán a pertenecer a la Enseñanza Media o Secundaria.

Para una mejor comprensión del comportamiento de la implementación de las diferentes bases curriculares originadas a partir de la última reforma educacional, acompañamos un cuadro cronológico.

ORD N $05 / 302$ del 14 de abril 2010

28 de enero de 2012. 
Tabla 1. Secuencia de bases curriculares según nivel

\begin{tabular}{|c|c|c|c|c|c|c|c|c|c|c|}
\hline & & & & $\overline{G R}$ & $\overline{O O}$ & & & & & \\
\hline AÑO & $\mathbf{1}^{\circ}$ & $2^{\circ}$ & $3^{\circ}$ & $4^{\circ}$ & $5^{\circ}$ & $6^{\circ}$ & $7^{\circ}$ & $8^{\circ}$ & LEY & BASES CURRICULARES \\
\hline 1997 & $\mathrm{X}$ & $\mathrm{X}$ & & & & & & & \multirow{17}{*}{ త్ర } & \multirow{15}{*}{ D.S. 40- 1996} \\
\hline 1998 & $\mathrm{X}$ & $\mathrm{X}$ & $\mathrm{X}$ & $\mathrm{X}$ & & & & & & \\
\hline 1999 & $X$ & $\mathrm{X}$ & $\mathrm{X}$ & $\mathrm{X}$ & $\mathrm{X}$ & & & & & \\
\hline 2000 & $\mathrm{X}$ & $\mathrm{X}$ & $\mathrm{X}$ & $\mathrm{X}$ & $\mathrm{X}$ & $\mathrm{X}$ & & & & \\
\hline 2001 & $X$ & $\mathrm{X}$ & $\mathrm{X}$ & $X$ & $\mathrm{X}$ & $\mathrm{X}$ & $\mathrm{X}$ & & & \\
\hline 2002 & $\mathrm{X}$ & $\mathrm{X}$ & $\mathrm{X}$ & $\mathrm{X}$ & $\mathrm{X}$ & $\mathrm{X}$ & $\mathrm{X}$ & $\mathrm{X}$ & & \\
\hline 2003 & $X$ & $\mathrm{X}$ & $\mathrm{X}$ & $X$ & $\mathrm{X}$ & $\mathrm{X}$ & $\mathrm{X}$ & $\mathrm{X}$ & & \\
\hline 2004 & $\mathrm{X}$ & $\mathrm{X}$ & $\mathrm{X}$ & $X$ & $\mathrm{X}$ & $\mathrm{X}$ & $\mathrm{X}$ & $\mathrm{X}$ & & \\
\hline 2005 & $X$ & $\mathrm{X}$ & $\mathrm{X}$ & $X$ & $\mathrm{X}$ & $\mathrm{X}$ & $\mathrm{X}$ & $\mathrm{X}$ & & \\
\hline 2006 & $\mathrm{X}$ & $\mathrm{X}$ & $\mathrm{X}$ & $\mathrm{X}$ & $\mathrm{X}$ & $\mathrm{X}$ & $\mathrm{X}$ & $\mathrm{X}$ & & \\
\hline 2007 & $\mathrm{X}$ & $\mathrm{X}$ & $\mathrm{X}$ & $X$ & $\mathrm{X}$ & $\mathrm{X}$ & $\mathrm{X}$ & $\mathrm{X}$ & & \\
\hline 2008 & $X$ & $\mathrm{X}$ & $\mathrm{X}$ & $X$ & $\mathrm{X}$ & $\mathrm{X}$ & $\mathrm{X}$ & $\mathrm{X}$ & & \\
\hline 2009 & $X$ & $\mathrm{X}$ & $\mathrm{X}$ & $\mathrm{X}$ & $\mathrm{X}$ & $\mathrm{X}$ & $\mathrm{X}$ & $\mathrm{X}$ & & \\
\hline 2010 & $X$ & $\mathrm{X}$ & $\mathrm{X}$ & $\mathrm{X}$ & $\mathrm{X}$ & $\mathrm{X}$ & $\mathrm{X}$ & $\mathrm{X}$ & & \\
\hline 2011 & $X$ & $\mathrm{X}$ & $\mathrm{X}$ & $\mathrm{X}$ & & & & & & \\
\hline 2011 & & & & & $\mathrm{X}$ & $\mathrm{X}$ & $\mathrm{X}$ & $\mathrm{X}$ & & \multirow{2}{*}{$\begin{array}{l}\text { D.S. } 256 \text { - } 2009 \\
\text { "AJUSTE CURRICULAR" }\end{array}$} \\
\hline 2012 & & & & & $\mathrm{X}$ & $\mathrm{X}$ & $\mathrm{X}$ & $\mathrm{X}$ & & \\
\hline 2012 & $\mathrm{X}$ & $\mathrm{X}$ & $\mathrm{X}$ & ¿? & & & & & \multirow{2}{*}{ LGE } & \multirow{2}{*}{ D.S. 439 - 2012} \\
\hline 2013 & $X$ & $\mathrm{X}$ & $\mathrm{X}$ & $\mathrm{X}$ & $\mathrm{X}$ & $\mathrm{X}$ & & & & \\
\hline 2013 & & & & & & & $\mathrm{X}$ & $\mathrm{X}$ & LOCE & D.S. $256-2009$ \\
\hline
\end{tabular}

Fuente: elaboración propia.

Otra cuestión no menor es la celeridad con que se implementaron las Bases Curriculares, si se tiene presente la experiencia anterior. La LOCE fue promulgada el 10 de marzo de 1990 y el Marco Curricular para la Enseñanza Básica del Decreto Supremo 40, seis años después; las problemáticas y vicisitudes de generar Bases Curriculares, en particular la que acabamos de señalar, están claramente evidenciadas en un artículo de un actor privilegiado, Cristian $\mathrm{Cox}^{3}$, quien en un claro artículo habla del "Marco curricular de Educación Básica: Arena de generación restringida" (2006: 10).

Finalmente, debemos señalar que un componente del Ajuste Curricular, los llamados Mapas de Progreso, que cada día ganaban más adeptos entre los docentes, fueron eliminados del Currículo de Enseñanza Básica con un escueto comunicado oficial de la unidad de

Coordinador Nacional del MECE, Primer Coordinador de la Unidad de Currículum y Evaluación. Uno de los gestores principales de la Reforma Educacional de los 90. 
Currículum y Evaluación, publicada en su página institucional ${ }^{4}$. En síntesis, se aduce que este componente no corresponde a los requerimientos de la LGE. Nosotros no tenemos ningún interés en debatir con la Unidad de Currículum y Evaluación, pero debemos señalar que este componente tampoco aparecía en la LOCE, pero las autoridades de la época los estimaron pertinentes.

Si bien es cierto nuestro interés es analizar y caracterizar el actual Currículo para la Enseñanza Básica chilena, lo expresado hasta este momento no tiene otra intención que no sea advertir la ocurrencia de algunas situaciones que, a nuestro parecer, necesariamente afectan el desarrollo del currículo y su instalación, las que eventualmente podrían ser dignas de discusión y análisis.

Sin desconocer la importancia de la problemática ya planteada, en lo que sigue nos abocaremos a desarrollar dos temáticas que subyacen en el núcleo de las Bases y que hacen necesaria una decodificación curricular, toda vez que no hay duda que están consideradas implícitamente, pero por su trascendencia se estima conveniente profundizar en ellas. Nos referimos al concepto de competencia y el currículo centrado en la enseñanza. Nos interesa dilucidar en qué medida estos dos conceptos inciden en el Marco Curricular, así como las eventuales consecuencias de no decodificarlos adecuadamente.

\section{2. ¿CAPACIDADES, COMPETENCIAS O HABILIDADES?}

Como es sabido, la LOCE traía aparejada un nuevo enfoque curricular, al introducir abruptamente conceptos bastante alejados del vocabulario habitual de los docentes en servicio en la Enseñanza Básica y Media, sin transición de objetivos generales, específicos y contenidos; se habla de objetivos fundamentales, contenidos mínimos obligatorios y posteriormente de aprendizajes esperados e indicadores de desempeño. Para implementar este nuevo currículum el MINEDUC introdujo recursos millonarios al sistema, desarrollando ingentes esfuerzos materiales y humanos para capacitar al profesorado conforme a la nueva visión educativa. Este fenómeno de la nueva nomenclatura no es menor, ya que el propio Cox escribió varios artículos y dictó innumerables charlas resaltando lo que él llamaba los "Seis equívocos de la Reforma Educacional", de hecho, en un artículo de su autoría escrito a cinco años de la instauración de la reforma, manifestaba: "Se observa que no hay una apropiación adecuada de la tríada conocimientos-habilidades-valores" (2001: 1).

Las bases definían correctamente los conceptos involucrados, se daban progresivamente las capacitaciones, pero los equívocos persistían y posiblemente aún persisten. ¿Por qué? Sencillamente el enfoque se contradice con la cultura curricular existente. Algo parecido podría volver a ocurrir con el actual Marco Curricular, pues, como se dijo, también conlleva una nueva nomenclatura muy distinta a la hasta ahora vigente ${ }^{5}$.

En las postrimerías de la década del 90 es invitado a nuestro país el catedrático español Martiniano Román, promoviendo el método T de planificación, de su autoría. Según su opinión, nuestra reforma tenía como leitmotiv el desarrollo de capacidades (según él las competencias correspondían al mundo del trabajo), entendidas como una habilidad general cuyo componente principal es cognitivo, acto seguido, planteaba un modelo donde definía

13 de noviembre de 2012.

De Objetivos Fundamentales y Contenidos Mínimos Obligatorios, ahora existen solamente Objetivos de Aprendizaje. 
el concepto de capacidad en función de destrezas y habilidades, asimilándolo al sistema métrico decimal, hablando del "sistema métrico mental" donde el metro es a una capacidad, la destreza al decímetro y la habilidad a un centímetro.

Martiniano Román recomendaba que cada establecimiento educacional debería declarar todas las capacidades que desarrollaría a lo largo de la enseñanza en un Panel de Capacidades, considerándose que en un año lectivo no era posible desarrollar más de cuatro capacidades.

Figura 1. Concepto de capacidad

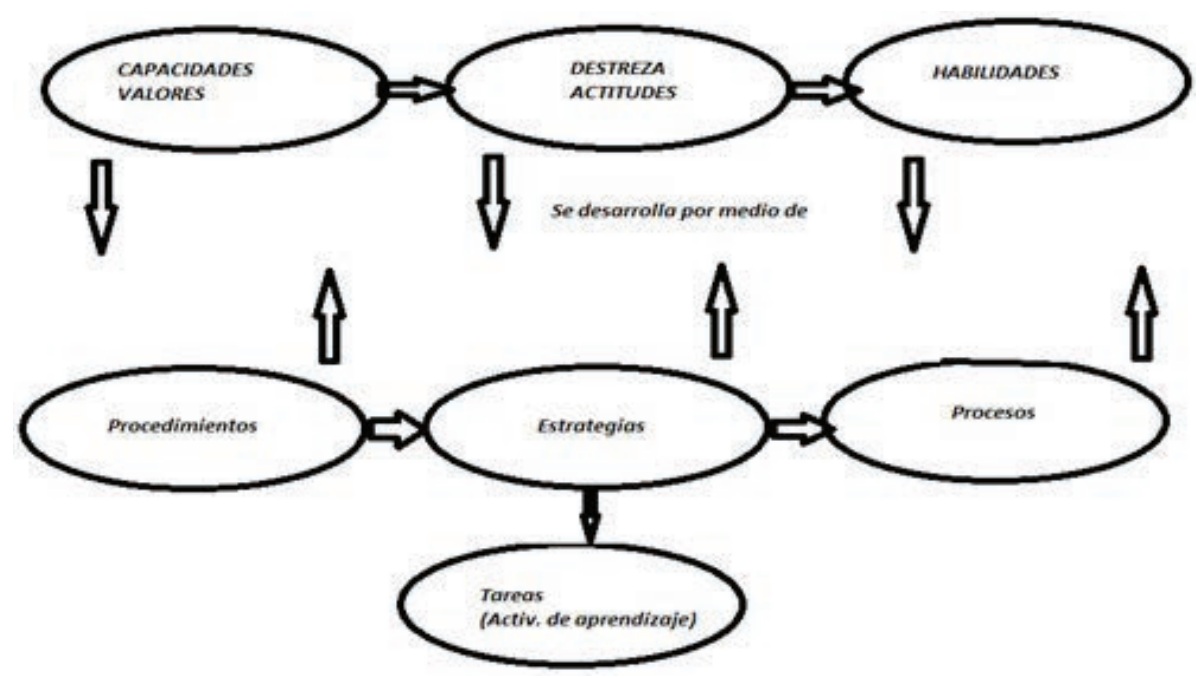

Fuente: Román y Díez (1998).

Una característica del Ajuste Curricular (MINEDUC, 2009) es que vuelve a definir, con mayor precisión, los numerosos conceptos que lo componen. Entre otros estipula que los Objetivos Fundamentales (OF) son los aprendizajes que los alumnos y las alumnas deben lograr al finalizar los distintos niveles de la Educación Básica y Media; que los Contenidos Mínimos Obligatorios (CMO) explicitan los conocimientos, habilidades y actitudes implicadas en los OF, y que el proceso de enseñanza debe convertir oportunidades de aprendizaje para cada estudiante con el fin de lograr los Objetivos Fundamentales. Si los OF están formulados desde la perspectiva del aprendizaje que cada alumno y alumna debe lograr, los CMO lo están desde la perspectiva de lo que cada docente debe obligatoriamente enseñar. También introduce el concepto de "instrumento curricular" a los recursos tales como: Marco Curricular, Programas, Planes y Textos de Estudio, SIMCE y los Mapas de Progreso, los que según se dijo, fueron eliminados para la Enseñanza Básica.

Una cuestión relevante y que ilumina nuestra discusión es que incluye el concepto de competencia (tomado de la OCDE) que debe ser entendido en el currículo chileno, lo que no es menor por la polisemia del concepto, por ejemplo, en Gutiérrez (2007) es posible encontrar 30 acepciones del término, y Cabrerizo, Rubio y Castillo (2008) aportan 15 definiciones. 
El Marco Curricular del Ajuste expresa:

Los OF-CMO del marco curricular están orientados al desarrollo de competencias que se consideran fundamentales para el desarrollo personal y para desenvolverse en el ámbito social, laboral y ciudadano. Siguiendo una definición de amplio consenso sobre las competencias, éstas aluden a la capacidad para responder a las exigencias individuales o sociales para realizar una tarea $\mathrm{o}$, dicho de otra forma, corresponden a la capacidad de articular y movilizar recursos aprendidos -saberes- con vistas a un desempeño de excelencia (MINEDUC, 2009: 10).

Fin de la discusión; por lo menos en $7^{\circ}$ y $8^{\circ}$ Básico, el proceso educativo debe orientarse hacia el desarrollo de competencias.

Cabe destacar, como suele suceder en las ciencias sociales, que frecuentemente se recurre a definiciones circulares (inadmisibles en la ciencias exactas) que tienen el grave problema de que la definición se sustenta en otros conceptos no definidos previamente, en este caso, la comprensión de la definición descansa en el hecho de que se debe tener claro qué es capacidad, el cual también es un concepto polisémico.

Este fenómeno de optar por el enfoque orientado de capacidades o competencias en el contexto de marcos curriculares no es extraño. Por ejemplo, en España en los últimos cincuenta años han promulgado cuatro leyes de educación, donde la Ley Orgánica de Ordenación General del Sistema Educativo Español (LOGSE), formulada en 1996, propugnaba el desarrollo de capacidades; por su parte, la Ley Orgánica de Educación (LOE), dictaminada en 2006, estipulaba el desarrollo de competencias. En el caso chileno, en los últimos veinte años se han dictado dos leyes educacionales, y para la Enseñanza Básica tres marcos curriculares, donde el D. 40 y sus diferentes modificaciones promueven el desarrollo de habilidades de orden superior. Como ya se dijo, el ajuste curricular del 2009 se refiere al desarrollo de competencias.

No obstante, nos enfocaremos ahora en lo que más interesa: ¿en qué perspectiva se encuentran las actuales Bases Curriculares (2012)? La respuesta es muy simple. En primer lugar, digamos que la introducción del decreto es un documento de 23 páginas, donde la palabra competencia no aparece y la palabra capacidad es citada catorce veces, sin embargo, el documento en esta materia es taxativo: el foco de estas bases se sitúa en lo que los estudiantes deben aprender, en términos de habilidades, actitudes y conocimientos (MINEDUC, 2012b: 7).

Esta triada, ya aludida anteriormente al citar a Cox (2006, 2001), es definida específicamente en el documento:

- Las habilidades son capacidades para realizar tareas y para solucionar problemas con precisión y adaptabilidad. Una habilidad puede desarrollarse en el ámbito intelectual, psicomotriz, afectivo y/o social.

- Los conocimientos corresponden a conceptos, redes de conceptos e información sobre hechos, procesos, procedimientos y operaciones. La definición contempla el conocimiento como información (sobre objetos, eventos, fenómenos, símbolos) y como comprensión, es decir, la información integrada en marcos explicativos e interpretativos mayores, que dan base para discernimiento y juicios.

- Las actitudes son disposiciones aprendidas para responder, de un modo favorable o no 
favorable, frente a objetos, ideas o personas; incluyen componentes afectivos, cognitivos y valorativos que inclinan a las personas a determinados tipos de acciones.

- Los conocimientos, las habilidades y las actitudes se abordan en estas Bases Curriculares de forma integrada. A la vez, para cada asignatura se destaca y secuencia de manera explícita las habilidades que le son propias, y las actitudes y los valores relacionados con aquellos Objetivos de Aprendizajes Transversales del ciclo que se prestan especialmente para ser desarrollados en el contexto de esa asignatura (MINEDUC, 2012b: 12).

Estos antecedentes nos llevan a una fase crucial de nuestro análisis, ¿qué tipo de currículum se debe adoptar? Nuevamente obtenemos una respuesta taxativa:

Los Objetivos de Aprendizaje relacionan en forma más explícita a las habilidades, los conocimientos y las actitudes y evidencian en forma clara y precisa cuál es el aprendizaje que el estudiante debe lograr. Se conforma así un currículum centrado en el aprendizaje, que declara explícitamente cuál es el foco del quehacer educativo (MINEDUC, 2012b: 3).

\section{3. ¿UN CURRÍCULUM CENTRADO EN EL APRENDIZAJE?}

Referirse a la mala calidad de la educación chilena, en todos sus niveles y sistemas, al margen de aparecer como un lugar común es casi de buen tono, por los dividendos que se le pueden extraer dependiendo de qué interlocutor se trata. Desde una mirada positiva, obviamente, como todo problema complejo, su explicación es de origen multifactorial. Posiblemente el factor que más incide podría ser la referencia a los modelos curriculares centrados en el profesor o en los contenidos como consecuencia de la formación de profesores basados en paradigmas meramente reproductores de saberes culturales, dirigidos a alumnos pasivos, acríticos acumuladores de información, sin tener en cuenta las diferencias individuales. La deficiencia de cada sistema educacional muestra sus falencias en el nivel inmediatamente superior y, finalmente, lo más grave queda expuesto en el mundo del trabajo y en la plena incorporación a la sociedad. También es cierto que las universidades en los últimos años, y con el objeto de enfrentar la realidad descrita, con distintos grados de profundidad están intentando derivar hacia un currículo basado en el desarrollo de competencias acordes con las necesidades que la sociedad actual demanda.

¿Ayudará un currículum centrado en el aprendizaje (CCA) a cambiar el actual estado de cosas? Previamente deberemos caracterizar lo que se debe entender como tal, para arribar a una adecuada conclusión.

Una primera pista la obtenemos del Informe Delors (1996), documento que impactó profundamente a la comunidad académica mundial, su amplia difusión y discusión nos permite soslayar entrar en detalles, sin embargo, hay un elemento contenido en dicho informe que nos permite identificar un primer componente que necesariamente debería constituir un enfoque CCA. Nos referimos a la necesidad de aprender a lo largo de toda la vida, el informe cataloga esta competencia como la llave del siglo XXI, debido a que es una manera eficiente de vivir en un mundo cambiante y de una acumulación ilimitada de información. Esta competencia conlleva a otra, la de aprender a aprender.

Con estos elementos, estamos en condiciones en perfilar un CCA. Previamente se debe tener presente: 
a) El educador en este contexto debe cuestionar la práctica tradicional de entender el proceso educativo como la relación que se establece entre el enseñar y el aprender, como si se tratase de una relación de causa-efecto; el profesor enseña (trasmite) contenidos que deben ser aprendidos (memorizados) por el estudiante. Esta visión mecánica y reduccionista del proceso educativo está siendo sustituido -desde tiempo ya- por los bajos resultados obtenidos, por aquella que propone como objetivo educativo fundamental el de preparar a los estudiantes para que aprendan mejor los contenidos de los planes de estudio pero, adicionalmente, que aprendan los procedimientos para que, dentro de la institución educativa y más allá de ella, puedan continuar aprendiendo, otorgando un mayor énfasis a los procesos cognitivos y a los procedimientos para aprender que a los contenidos -debido a su volumen y vigencia relativa-, obviamente, estas herramientas, conformadas por procesos y procedimientos cognitivos, habitualmente no se producen en el vacío, sino que están estrechamente vinculados a unos contenidos disciplinarios (Gutiérrez, 2003).

b) El educador deberá asumir un enfoque preferentemente constructivista, orientado hacia el aprendizaje significativo ausbeliano y el concepto vigostskyano de Zona de Desarrollo Próximo (ZDP).

c) El educador debe propender a explotar al máximo el Potencial de Aprendizaje $(\mathrm{PA})^{6}$ del aprendiz.

Para mayor claridad mostramos un esquema que integra los principales componentes del modelo, según nuestra visión. A continuación, resumimos el modelo.

Como ya se dijo en un enfoque centrado en el aprendizaje el destinatario final es la persona individual que aprende, ya que el aprendiz debe desarrollar sus capacidades de aprendizaje a partir de su propio potencial de aprendizaje, de modo que pueda comprender el mundo, reflexionar de forma crítica sobre los diversos acontecimientos, y actuar de manera eficaz ante los problemas.

El proceso tradicional de enseñanza-aprendizaje centrado en la transmisión de información y en la figura del profesor como fuente casi única del saber, debe derivar, tal como lo prescriben Feurestein y Vygotsky, en un profesor mediador entre el que aprende y lo que se debe aprender, para, entre otras cosas, permitir la autonomía del aprendiz; en este enfoque el rol del profesor cambia drásticamente. La información, sus fuentes y los medios para difundirla se han diversificado de tal manera como consecuencia del avance científico tecnológico y el desarrollo de las nuevas tecnologías de la comunicación y la información, que puede hablarse de la emergencia de un nuevo paradigma educativo.

Gutiérrez (2003), después de un profundo análisis, llega a la conclusión de que un enfoque curricular centrado en el aprendizaje debe sustentarse en una visión constructivista, por la calidad de los aprendizajes y las estrategias que se deben lograr deben ser altamente significativos para el que aprende, lo que se adapta bastante bien con los conceptos de aprendizaje significativo de Ausubel y lo referente al conocimiento previo, frecuentemente confundido con la idea de diagnóstico. Otro concepto crucial es el de Zona de Desarrollo Próximo de Vygotsky, concepto que claramente evidencia el carácter mediador del profesor.

El "Potencial de Aprendizaje" (PA) es uno de los conceptos más prometedores como complemento y/o sustituto del estático concepto de inteligencia y su medida. Basada en el concepto vygostkyano de la ZDP, así como en otros más recientes como "modificabilidad" o "educabilidad", en los últimos años se ha desarrollado una serie de investigaciones tratando tanto de profundizar en este concepto, como de proceder a su medida (Fernández-Ballesteros, 1989). 
Figura 2. Currículum centrado en el aprendizaje

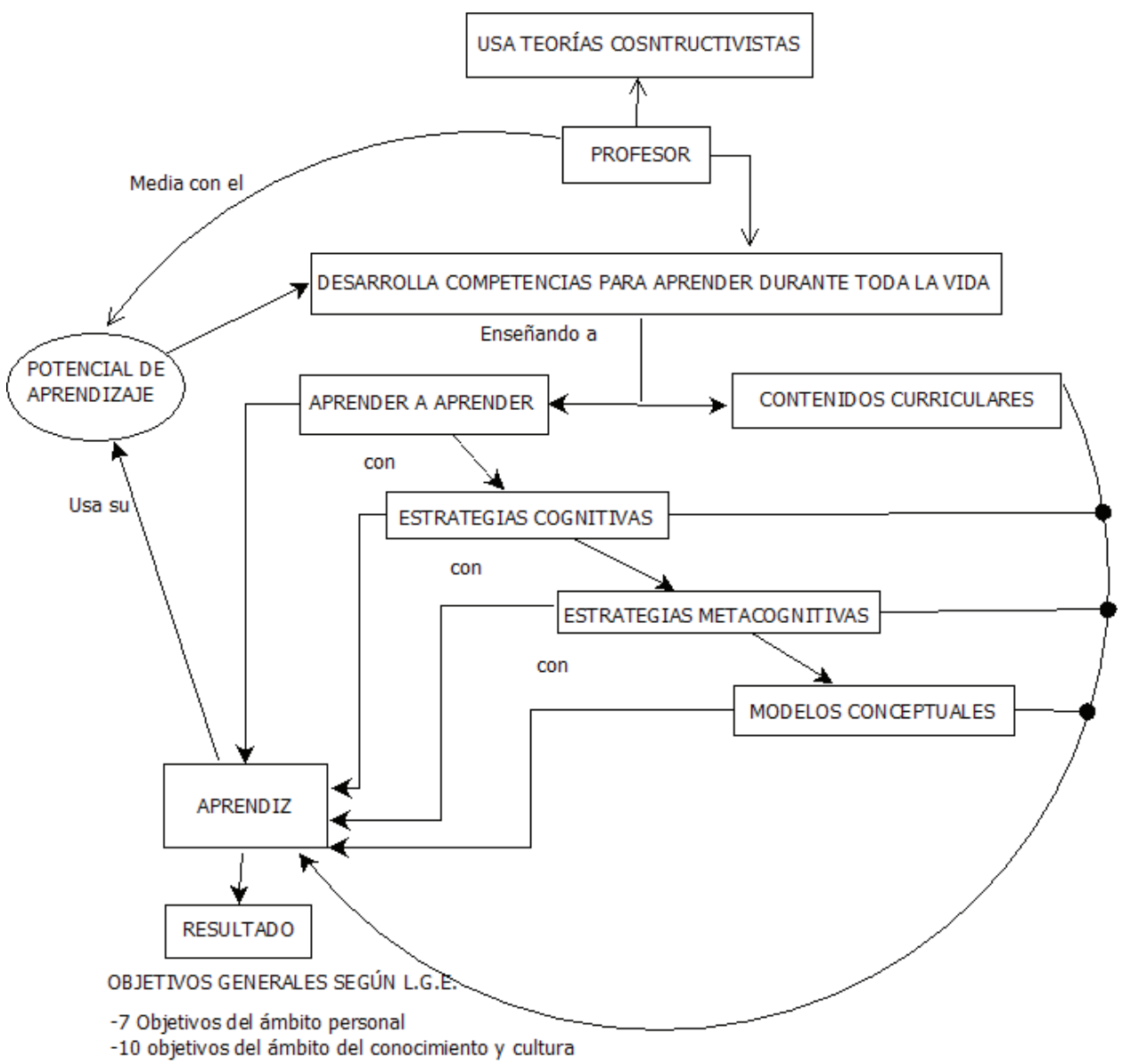

Fuente: elaboración propia.

La enseñanza para toda la vida se obtiene aprendiendo a aprender. Para Román y Díez (1998), aprender a aprender implica:

a) Aprendizaje y uso de estrategias cognitivas entendidas como el procedimiento más adecuado para adquirir un conocimiento.

b) Aprendizaje y uso de estrategias metacognitivas, entendidas como darse cuenta de los propios procesos del pensar, para conocerlos y mejorarlos.

c) Aprendizaje y uso de modelos conceptuales que son formas de representación cognitiva.

Los autores utilizan variados criterios para clasificar las estrategias cognitivas. A continuación se muestra en forma resumida la clasificación de Beltrán et al., (1987: 134). 
- Búsqueda de información:

o Selección adecuada de fuentes.

o Hacer preguntas adecuadas.

o Uso adecuado de la biblioteca.

o Uso adecuado del material de referencia.

- Asimilar y retener información:

o Saber escuchar para comprender.

o Cómo estudiar bien.

o Cómo generar representaciones.

o Leer comprensivamente.

o Saber tomar registros.

- Organizar el aprendizaje:

o Saber priorizar.

o Programación del tiempo.

- Desarrollar la creatividad:

o Desarrollo de actividades inquisitivas.

o Razonar inductivamente.

o Uso de analogías.

o Desarrollo de la flexibilidad.

- Desarrollar competencia analítica:

o Actitud crítica.

o Razonamiento deductivo.

o Evaluación de ideas e hipótesis.

- La toma de decisiones:

o Identificación de alternativas.

o Cómo hacer elecciones racionales.

- Habilidades sociales:

o Cómo evitar conflictos interpersonales.

o Cómo cooperar y obtener cooperación

o Cómo motivar a otros.

En cuanto a las habilidades metacognitivas, según Gutiérrez (2003) se deberían desarrollar habilidades tales como:

- Conocimiento sobre los propios hábitos y habilidades de estudio.

- Selección de estrategias de aprendizaje adecuadas a determinadas tareas de estudio.

- Capacidad de verificar los resultados del propio comportamiento de estudio.

- Tendencia al uso de estrategias compensatorias o alternativas cuando el resultado no es exitoso.

En cuanto a los modelos conceptuales, Román y Díez (1998) expresan que cada uno de nosotros en nuestro cerebro lleva uno o varios modelos del universo y lo que hay en su interior. También llevamos nuestro propio modelo conceptual, el que elaboramos a lo largo de muchos años, aglutinándose con tres elementos básicos:

a) Percepción organizada: donde la experiencia sensorial es la principal fuente de información.

b) Una representación que trata de globalizar lo percibido: una suerte de organizadores gráficos. 
c) Una conceptualización: unas veces conocemos la realidad porque la percibimos, otras porque la representamos, y de ordinario porque la conceptualizamos y tenemos una opinión de ella. Es en este momento cuando la realidad externa forma parte de nuestra realidad personal y cognitiva. Los mapas conceptuales son instrumentos adecuados para pasar del hecho al concepto o del concepto al hecho; en general: redes, esquemas, marcos y mapas conceptuales son útiles para significar conceptos y su aprendizaje.

Finalmente, el resultado esperado conforme al artículo 29 de la Ley General de Educación está conformado por estos 17 objetivos generales, los que deben ser adecuadamente interpretados por las respectivas Bases Curriculares:

\section{En el ámbito personal y social:}

a. Desarrollarse en los ámbitos moral, espiritual, intelectual, afectivo y físico de acuerdo a su edad.

b. Desarrollar una autoestima positiva y confianza en sí mismos.

c. Actuar de acuerdo con valores y normas de convivencia pacífica, conocer sus derechos y responsabilidades, y asumir compromisos consigo mismo y con los otros.

d. Reconocer y respetar la diversidad cultural, religiosa y étnica y las diferencias entre las personas, así como la igualdad de derechos entre hombres y mujeres, y desarrollar capacidades de empatía con los otros.

e. Trabajar individualmente y en equipo, con esfuerzo, perseverancia, responsabilidad y tolerancia a la frustración.

f. Practicar actividad física adecuada a sus intereses y aptitudes.

g. Adquirir hábitos de higiene y cuidado del propio cuerpo y salud.

\section{En el ámbito del conocimiento y la cultura:}

a. Desarrollar la curiosidad, la iniciativa personal y la creatividad.

b. Pensar en forma reflexiva, evaluando y utilizando información y conocimientos, de manera sistemática y metódica, para la formulación de proyectos y resolución de problemas.

c. Comunicarse con eficacia en lengua castellana, lo que implica comprender diversos tipos de textos orales y escritos adecuados para la edad y expresarse correctamente en forma escrita y oral.

d. Acceder a información y comunicarse usando las tecnologías de la información y la comunicación en forma reflexiva y eficaz.

e. Comprender y expresar mensajes simples en uno o más idiomas extranjeros.

f. Comprender y utilizar conceptos y procedimientos matemáticos básicos, relativos a números y formas geométricas, en la resolución de problemas cotidianos, y apreciar el aporte de la matemática para entender y actuar en el mundo.

g. Conocer los hitos y procesos principales de la historia de Chile y su diversidad geográfica, humana y sociocultural, así como su cultura e historia local, valorando la pertenencia a la nación chilena y la participación activa en la vida democrática.

h. Conocer y valorar el entorno natural y sus recursos como contexto de desarrollo humano y tener hábitos de cuidado del medioambiente.

i. Aplicar habilidades básicas y actitudes de investigación científica, para conocer y com- 
prender algunos procesos y fenómenos fundamentales del mundo natural, y de aplicaciones tecnológicas de uso corriente.

j. Conocer y apreciar expresiones artísticas de acuerdo a la edad y expresarse a través de la música y las artes visuales.

\section{4. ¿NADA NUEVO BAJO EL SOL?}

Como puede verificarse en el presente trabajo, inicialmente hemos entregado antecedentes que describen la secuencia con que se ha llegado a las actuales Bases Curriculares para la Enseñanza Básica, y sus eventuales consecuencias negativas para su implementación. Luego se ha analizado la evolución e instalación del concepto de competencia, para concluir que, en la actualidad, para mantener la debida coherencia con el Marco Curricular para $7^{\circ}$ y $8^{\circ}$ Básico y hasta el año 2017, se deberán desarrollar competencias; paralelamente desde $1^{\circ}$ a $6^{\circ}$ deben desarrollarse habilidades tal como lo prescribe el marco correspondiente. Este "doble estándar" también puede aportar ruido a la implementación de las Bases Curriculares. Nuestro punto central ha sido el enfoque curricular centrado en el aprendizaje, donde hemos detallado completamente lo que, a nuestro entender, debería entenderse como tal a la hora de implementarlo.

A modo de conclusión nos abocaremos a extraer algunas consideraciones, teniendo como fondo precisamente los Marcos Curriculares que se han prescrito los últimos 20 años. Iniciamos nuestro análisis citando a Rosas y Sebastián:

En gran parte de los países hispanoparlantes, influidos por la reforma educativa española, la educación es explícitamente constructivista, un buen ejemplo de ello es el decreto 40 de Chile, que establece que: "Al mismo tiempo, orienta al nuevo marco curricular el principio de que el aprendizaje debe tener lugar en una nueva forma de trabajo pedagógico, que tiene por centro la actividad de los alumnos, sus características y conocimientos previos. Centrar el trabajo pedagógico en el aprendizaje más que en la enseñanza exige, adicionalmente, desarrollar estrategias pedagógicas diferenciadas y adaptadas a los distintos ritmos y estilos de aprendizaje de un alumnado heterogéneo, y reorientar el trabajo escolar desde su forma actual, predominantemente lectiva, a una basada en actividades de exploración, búsqueda de información y construcción de nuevos conocimientos por parte de los alumnos, tanto individual como colaborativamente y en equipo. Por último, el aprendizaje buscado se orienta en función del desarrollo de destrezas y capacidades de orden superior (tales como descripción, clasificación, análisis, síntesis, capacidad de abstracción, y otras especificadas en cada sección de los Objetivos Fundamentales), a través del conocimiento y dominio de unos contenidos considerados esenciales para constituir el núcleo cultural común de las nuevas generaciones del país" (Ministerio de Educación de Chile, 1992). Más allá de la discusión acerca de si es legítimo o siquiera conveniente dejar expresada una corriente teórica en un cuerpo legal (2008: 7).

De la lectura de este texto pueden extraerse cuatro características del Marco Curricular provenientes de la LOCE:

a) Es constructivista según Ausubel.

b) El currículum está centrado en el alumno. 
c) Cambia desde el paradigma enseñanza-aprendizaje al de aprendizaje-enseñanza.

d) Busca el desarrollo de destrezas y capacidades de orden superior -competencias/capacidades-.

Es interesante resaltar que este texto se mantuvo en las modificaciones del D. 232 de 2002, el que modificó parcialmente el citado decreto 40. Es necesario tener presente que en el texto que estamos analizando se dice expresamente: el principio de que el aprendizaje debe tener lugar en una nueva forma de trabajo pedagógico. Como así mismo: centrar el trabajo pedagógico en el aprendizaje más que en lo que la enseñanza exige, adicionalmente, desarrollar estrategias pedagógicas diferenciadas y adaptadas a los distintos ritmos. De lo anterior, se espera de los docentes una nueva forma de trabajo siendo ello un principio, exigiendo que el trabajo pedagógico deba centrarse en el aprendizaje. En un texto similar con ligeros cambios en la redacción se prescribe algo parecido en el Ajuste Curricular del $2009^{7}$.

¿A dónde pretendemos llegar? Sencillamente a que el currículum centrado en el aprendizaje esté prescrito en el "currículum oficial" desde el Decreto Supremo de Educación $\mathrm{N}^{\circ} 40$ de 1996. ¡16 años! Sin embargo, en general la docencia en el aula es eminentemente expositiva, contenidista como diría Cox $(2006,2001)$.

Por los antecedentes expuestos, creemos que una manera de mejorar la calidad de la Enseñanza Básica es canalizar los esfuerzos para asegurar que el profesorado se apropie adecuadamente de estas Bases Curriculares, se convenza de las ventajas metodológicas al asumir integralmente un currículo centrado en el aprendizaje, tal como aquí se ha descrito. Y que, por otra parte, los expertos direccionen sus objetos de investigación hacia las representaciones que tienen los docentes en el trabajo de aula, y sus posturas frente al Currículum Centrado en el Aprendizaje.

\section{REFERENCIAS BIBLIOGRÁFICAS}

Beltrán, J., García-Alcañiz, E., Moraleda, M. G., Calleja, F. y Santiuste, V. (1987). Piscología de la educación. Madrid: Eudema.

Cabrerizo, J., Rubio, M. y Castillo, S. (2008). Programación por competencias. Madrid: Pearson Educación.

Cox, C. (2006). Construcción política de reformas curriculares: El caso de Chile en los 90. Profesorado. Revista de Currículum y Formación del Profesorado, vol. 10, n. 1, 1-24.

(2001). Seis equívocos de la Reforma Eduacional. Santiago: Documento de apoyo, Unidad de Currículum y Evaluación, MINEDUC.

Delors, J. (1996). La Educación encierra un tesoro. París: Ediciones UNESCO.

Fernández-Ballesteros, R. (1989). Potencial de aprendizaje: Una presentación. Estudios de Psicología, n. 38, 62-68.

Gutiérrez, J. (2007). Diseño curricular basado en competencias. Santiago: Altazor.

Gutiérrez, O. (2003). Enfoques y modelos educativos centrados en el aprendizaje. Recuperado de http://www.lie.upn.mx/docs/docinteres/EnfoquesyModelosEducativos3.pdf, consultado en febrero de 2013.

MINEDUC. (2012a). Nuevas Bases Curriculares 2012 para la Enseñanza Básica. Santiago: MINEDUC.

Desde 1990 los Ministros de Educación se han cambiado en un promedio de 1,7 por año, implicando rotaciones de equipos e inestabilidad de las políticas educativas. 
(2012b). Bases Curriculares. 2012 Educación Básica. Santiago: MINEDUC.

. (2009). Objetivos Fundamentales y Contenidos Mínimos Obligatorios de la Educación Básica y Media. Santiago: MINEDUC.

Román, P. y Díez, E. (1998). Aprendizaje y curriculum (5 Ed.). Santiago: FIDE.

Rosas, R. y Sebastián, Ch. (2008). Piaget, Vigotsk y Maturana: Constructivismo a tres voces (2a Ed.). Buenos Aires: AIQUE. 